\title{
CD5-positive follicular lymphoma: clinicopathologic correlations and outcome in 88 cases
}

Yu Li ${ }^{1,2}$, Shimin $\mathrm{Hu}^{1}$, Zhuang Zuo ${ }^{1}$, Ming Hong ${ }^{1}$, Pei Lin ${ }^{1}$, Shaoying $\mathrm{Li}^{1}$, Sergej Konoplev ${ }^{1}$, Zhen Wang ${ }^{1}$, Joseph D Khoury ${ }^{1}$, Ken H Young ${ }^{1}$, L Jeffrey Medeiros ${ }^{1}$ and C Cameron Yin $^{1}$

${ }^{1}$ Department of Hematopathology, UT MD Anderson Cancer Center, Houston, TX, USA and ${ }^{2}$ Department of Pathology, Chongqing Medical University, Chongqing, China

Follicular lymphoma is a low-grade B-cell lymphoma of germinal center B-cell origin that typically lacks CD5 expression. We describe the clinicopathologic features of 88 cases of CD5+ follicular lymphoma (53 men, 35 women; median age, 60 years; range, 31-86). Follicular lymphoma was diagnosed initially in lymph nodes in 66 and extranodal sites in 22 patients. Eighty-one patients had lymphadenopathy, 66 had more than one involved site, 46 had bone marrow involvement, and 7 had splenomegaly. Staging information was available for 84 patients: 52 stage IV, 18 stage III, 12 stage II, and 2 stage I. Sixty-one cases were grade 1 or 2 and 27 were grade 3. The median proliferation index (Ki-67) was 30\%. CD5 expression was detected by flow cytometry in 69 , immunohistochemistry in 8 , and both methods in 11 cases. The presence of $t(14 ; 18)(q 32 ; q 21) / / G H-B C L 2$ or other $B C L 2$ translocation was detected in $28 / 44(64 \%)$ cases. A total of $38(43 \%)$ patients also had diffuse large B-cell lymphoma, concurrent with $(n=20)$, subsequent to $(n=13)$, or developing before CD5+ follicular lymphoma $(n=5)$. All patients received chemotherapy; 12 also received stem-cell transplantation. With a median follow-up of 55 months (range, 0.5-207), 15 patients died, 46 were alive with disease, and 20 were in clinical remission. Compared with a matched group of patients with CD5- follicular lymphoma, patients with CD5+ follicular lymphoma more commonly had an International Prognostic Index $>2(35 / 80$ vs $10 / 99, P<0.001)$, more often developed diffuse large B-cell lymphoma (38/88 vs 17/99; $P<0.001)$, and had a shorter median progression-free survival (44 vs 89 months, $P=0.0042$ ). Higher $\mathrm{Ki}-67$ and International Prognostic Index were identified as poor prognostic factors in both the groups. We conclude that CD5 expression in follicular lymphoma is associated with a higher International Prognostic Index, higher rate of transformation to diffuse large B-cell lymphoma, and shorter progression-free survival.

Modern Pathology (2015) 28, 787-798; doi:10.1038/modpathol.2015.42; published online 6 March 2015

Follicular lymphoma is the most common indolent non-Hodgkin lymphoma and the second most common non-Hodgkin lymphoma overall in the USA. ${ }^{1}$ Follicular lymphoma is defined as a neoplasm composed of germinal center-derived B-cells that usually form at least a partial follicular growth pattern. ${ }^{1}$ Most patients with follicular lymphoma present with a widespread disease and have an indolent clinical course. ${ }^{1}$ The genetic hallmark of follicular lymphoma, $\mathrm{t}(14 ; 18)(\mathrm{q} 32 ; \mathrm{q} 21) / I G H-B C L 2$, has been identified in $80-90 \%$ of cases and leads to deregulated expression of $B C L 2 .^{2}$

Correspondence: Dr CC Yin, MD, PhD, Department of Hematopathology, UT MD Anderson Cancer Center, Houston, TX 77030, USA.

E-mail: cyin@mdanderson.org

Received 12 November 2014; revised 20 January 2015; accepted 21 January 2015; published online 6 March 2015
Follicular lymphoma has a specific immunophenotype, positive for pan B-cell markers, monotypic surface immunoglobulin light chain, and follicular center cell-associated antigens, such as CD10, BCL-6 and LMO2, but negative for pan T-cell antigens. ${ }^{1}$ The T-cell marker CD5 is usually negative in follicular lymphoma and therefore CD5 expression is useful in distinguishing follicular lymphoma from other lowgrade B-cell lymphomas. ${ }^{3}$ However, cases of CD5+ follicular lymphoma have been reported in small numbers. ${ }^{4-9}$ More recently, Miyoshi et al ${ }^{10}$ described a series of 22 cases of CD5+ follicular lymphoma from Japan and reported that patients with CD5+ follicular lymphoma had a higher frequency of peripheral blood involvement and CD25 and MUM1 expression, a lower frequency of $\mathrm{t}(14 ; 18)(\mathrm{q} 32 ; \mathrm{q} 21)$, and the worse overall survival.

In this study, we describe the clinicopathologic, immunophenotypic, and molecular genetic 
features of 88 patients with CD5+ follicular lymphoma seen at our institution, the largest series reported to date.

\section{Materials and methods}

\section{Case Selection}

We searched the database of the Department of Hematopathology at The University of Texas MD Anderson Cancer Center, from 1 January 2000 to 30 June 2014, for cases of follicular lymphoma that were positive for CD5 as shown by flow cytometry immunophenotypic analysis or immunohistochemistry. A group of 99 patients with CD5-negative follicular lymphoma matched for age, gender, and stage were selected as a comparison group. The diagnosis was based on morphologic and immunophenotypic criteria as specified in the World Health Organization classification. ${ }^{1}$ Clinical information was obtained by review of medical records. This study was conducted according to an institutional review board-approved laboratory protocol.

\section{Morphologic Evaluation and Immunophenotypic Analysis}

We reviewed hematoxylin-eosin-stained tissue sections of diagnostic biopsy specimens. In patients with bone marrow involvement, hematoxylin-eosinstained core biopsy and clot sections, as well as Wright-Giemsa-stained aspirate smears and touch imprints were also reviewed.

Flow cytometry immunophenotypic analysis was performed on cell suspensions of tissue biopsy or bone marrow aspirate using a FACScan instrument (Becton-Dickinson Biosciences, San Jose, CA, USA) as described previously. ${ }^{11}$ The lymphocyte population was gated using right angle side scatter and CD45 expression. The panel of monoclonal antibodies included reagents specific for CD3, CD5, CD10, CD11c, CD19, CD20, CD22, CD23, CD30, CD38, CD43, CD45, CD79b, CD200, FMC-7, and surface immunoglobulin kappa and lambda light chains (Becton-Dickinson Biosciences).

Immunohistochemical studies were performed using formalin-fixed, paraffin-embedded sections of nodal or extranodal tissue biopsy specimens or bone marrow core biopsy or aspirate clot specimens using the avidin-biotin-peroxidase complex method and an automated immunostainer (Ventana-Biotech, Tucson, AZ, USA) as described previously. ${ }^{12}$ All tissue sections underwent heat-induced antigen retrieval. The antibodies used were specific for CD3, CD20, CD43, BCL-6, MUM1, and Ki-67 (Dako, Carpinteria, CA, USA); CD5 and cyclin D1 (SP4; Labvision/Neomarkers, Fremont, CA, USA); CD10, CD23, and BCL2 (Novocastra/Vision Biosystem, Benton Lane, Newcastle-upon-Tyne, UK); CD21 (Leica Biosystems, Buffalo Grove, IL, USA); and
PAX-5 (Transduction Labs, San Diego, CA, USA). Membranous reactivity for CD5 by the neoplastic CD20+ B-cells was considered positive. CD3+ T-cells were used for comparison in the assessment of CD5 expression.

\section{Cytogenetic Analysis}

Conventional cytogenetic analysis was performed on metaphase cells prepared form bone marrow aspirate or cell suspensions from tissue biopsy specimens using standard techniques. Twenty Giemsa-banded metaphases were analyzed, and the results were reported using the International System for Human Cytogenetic Nomenclature (2013).

Fluorescence in situ hybridization analysis for the detection of $I G H$ (or $I G L$ ) and BCL2 gene rearrangement was performed using a LSI IGH(IGL)/BCL2 dual-color, dual-fusion translocation probe on interphase nuclei obtained from bone marrow cells or tissue sections, according to the manufacturer's instructions (Vysis/Abbott Laboratories, Des Plaines, IL, USA). We also performed fluorescence in situ hybridization analysis for the presence of $I G H$ and CCND1 gene rearrangement using a LSI IGH/CCND1 dual-color, dual-fusion translocation probe in a sub set of the cases (Vysis/Abbott Laboratories).

\section{Molecular Analysis}

DNA was extracted from bone marrow aspirate or fixed, paraffin-embedded tissue and was subjected to TaqMan IGH/BCL2 qualitative real-time polymerase chain reaction coupled with fluorescent-based capillary electrophoresis (Applied Biosystems, Foster City, CA, USA), as described previously. ${ }^{13}$ Amplification of a $93 \mathrm{bp}$ sequence from the cyclophilin gene was performed simultaneously in all cases to normalize the IGH/BCL2 values to the amount of input DNA. DNA obtained from the HL60 cell line was used as a negative control.

\section{Statistical Analysis}

Statistical analyses were performed using the GraphPad Prism 5 and SAS-9. Overall survival was calculated from the date of initial diagnosis to the date of death or last follow-up. Progression-free survival was calculated from the date of initial diagnosis to the date of progression or last followup. Survival was analyzed using the Kaplan-Meier method and was compared using the log rank test. Fisher's exact test was utilized to analyze differences between the CD5+ and CD5- follicular lymphoma groups. Univariate Cox proportional hazards model analyses for overall survival and progression-free survival were performed. A $P$ value of $<0.05$ was considered statistically significant. 


\section{Results}

\section{Clinical Findings}

We identified 88 patients with CD5+ follicular lymphoma. This group was extracted from a total of $3286(2.7 \%)$ patients with follicular lymphoma seen at our institution during a 14.5-year study period. There were 53 men and 35 women with a median age of 60 years (range, 31-86 years) at time of diagnosis. The most common presenting symptom was lymphadenopathy, reported by 68 (82\%) patients. Five patients were found incidentally to have lymphadenopathy during a work-up for other diseases. In addition to lymphadenopathy, 4 patients had gastrointestinal tract symptoms, 3 had pleural effusions, 3 had leukocytosis, 3 had a scalp lesion, and 1 patient each had an orbital mass, lacrimal gland mass, parotid nodule, submandibular gland mass, tongue mass, breast mass, lung mass, periureteral nodule, or chest-wall mass.

At the time of presentation at our institution, 10 $(12 \%)$ patients had B-symptoms. Eighty-one (98\%) patients had lymphadenopathy, including 56 (68\%) with involvement of multiple lymph nodes, $5(6 \%)$ with single lymph node involvement, and 20 (24\%) with involvement of lymph node and extranodal tissue sites. Seven (8\%) patients had splenomegaly. Bone marrow was involved in 46 of 78 (59\%) cases assessed. Peripheral blood was involved in 12 of 29 $(41 \%)$ cases assessed by flow cytometry. Staging information was available in 84 patients, including 2 $(2 \%)$ patients with stage I, $12(14 \%)$ with stage II, 18 $(22 \%)$ with stage III, and $52(62 \%)$ with stage IV disease. The International Prognostic Index (IPI) was available in 80 cases: 2 patients had an IPI of 0,9 an IPI of 1, 34 an IPI of 2, 29 an IPI of 3, and 6 patients had an IPI of 4 . The Follicular Lymphoma IPI (FLIPI) was also available in 80 cases. The FLIPI scores were 0 in 4 patients; 1 in 21 patients; 2 in 31 patients; 3 in 17 patients, and 4 in 7 patients.

Laboratory data were available for 80 patients. Ten $(13 \%)$ patients had leukopenia and $6(8 \%)$ had leukocytosis (median leukocyte count, $5.35 \mathrm{k} / \mu \mathrm{l}$; range, $0.7-12.7 \mathrm{k} / \mu \mathrm{l}$; reference range, $4-11 \mathrm{k} / \mu \mathrm{l})$. Twenty-five $(31 \%)$ patients had absolute lymphopenia and one patient had absolute lymphocytosis (median absolute lymphocyte count, $1.77 \mathrm{k} / \mu \mathrm{l}$; range, $0.6-46.7 \mathrm{k} / \mu \mathrm{l}$; reference range, $1.0-4.8 \mathrm{k} / \mu \mathrm{l})$. Thirty-nine $(49 \%)$ patients had anemia (median hemoglobin, $12.9 \mathrm{~g} / \mathrm{dl}$; range, 8.7-15.9 g/dl; reference range, $14-18 \mathrm{~g} / \mathrm{dl}$ for men and $12-16 \mathrm{~g} / \mathrm{dl}$ for women). Twenty (25\%) patients had thrombocytopenia (median platelet count, $219 \mathrm{k} / \mu \mathrm{l}$; range, 17$442 \mathrm{k} / \mu \mathrm{l}$; reference range, $140-440 \mathrm{k} / \mu \mathrm{l}$ ). Fifteen of $78(19 \%)$ patients had an elevated serum lactate dehydrogenase (LDH) level (median, 566 IU/l; range, 341-1739 IU/l; reference range, 313-618 IU/l), and 49 of $75(65 \%)$ patients had an elevated level of serum $\beta 2$-microglobulin level (median, $2.7 \mathrm{mg} / \mathrm{l}$; range, $0.9-7.1 \mathrm{mg} / \mathrm{l}$; reference range, $0.7-1.8 \mathrm{mg} / \mathrm{l}$ ).
The clinical and laboratory findings are summarized in Table 1.

\section{Morphologic Findings}

Follicular lymphoma was initially diagnosed in lymph nodes in $66(75 \%)$ patients and in extranodal biopsy sites in $22(25 \%)$ patients. The extranodal sites included gastrointestinal tract $(n=4)$, salivary gland $(n=4)$, pleural fluid $(n=3)$, scalp $(n=3)$, orbit $(n=2)$, and 1 case each in lung, breast, tongue, lacrimal gland, chest-wall or periureteral soft tissue. Morphologically, the architecture of the lymph nodes was totally or partially replaced by lymphoma. Most cases $(n=61)$ showed a predominantly follicular pattern with closely packed follicles, 10 cases showed a mixed follicular and diffuse pattern, and 1 case was an axillary lymph node core needle biopsy specimen that showed a diffuse pattern with no follicular areas identified. Sixteen cases showed composite follicular lymphoma and another lymphoma including diffuse large B-cell lymphoma $(n=13)$, classical Hodgkin lymphoma $(n=2)$, and chronic lymphocytic leukemia/small lymphocytic lymphoma $(n=1)$. Within the follicles, a mixed

Table 1 Clinical and laboratory features of CD5+ follicular lymphoma

\begin{tabular}{|c|c|c|c|}
\hline Features & Presence & Total & Presence \% \\
\hline Age $>60$ years & 42 & 88 & 48 \\
\hline Male & 53 & 88 & 60 \\
\hline Female & 35 & 88 & 40 \\
\hline B-symptoms & 10 & 83 & 12 \\
\hline Lymphadenopathy & 81 & 83 & 98 \\
\hline Splenomegaly & 7 & 83 & 8 \\
\hline Nodal presentation & 66 & 88 & 75 \\
\hline Extranodal presentation & 22 & 88 & 25 \\
\hline $\mathrm{BM}$ involvement & 46 & 78 & 59 \\
\hline \multicolumn{4}{|l|}{ Stage } \\
\hline I & 2 & 84 & 2 \\
\hline II & 12 & 84 & 14 \\
\hline III & 18 & 84 & 21 \\
\hline IV & 52 & 84 & 62 \\
\hline \multicolumn{4}{|l|}{ IPI } \\
\hline $0-2$ & 45 & 80 & 56 \\
\hline $3-4$ & 35 & 80 & 44 \\
\hline \multicolumn{4}{|l|}{ FLIPI } \\
\hline $0-2$ & 56 & 80 & 70 \\
\hline $3-4$ & 24 & 80 & 30 \\
\hline $\mathrm{WBC}<4 \mathrm{k} / \mu \mathrm{l}$ & 10 & 80 & 13 \\
\hline $\mathrm{WBC}>11 \mathrm{k} / \mu \mathrm{l}$ & 6 & 80 & 8 \\
\hline ALC $<1000 / \mu \mathrm{l}$ & 25 & 80 & 31 \\
\hline $\mathrm{Hb}<12 \mathrm{~g} / \mathrm{dl}$ & 39 & 80 & 49 \\
\hline Platelet $<140 \mathrm{k} / \mu \mathrm{l}$ & 20 & 80 & 25 \\
\hline Elevated LDH & 15 & 78 & 19 \\
\hline Elevated $\beta 2 \mathrm{M}$ & 49 & 75 & 65 \\
\hline
\end{tabular}

Abbreviations: BM, bone marrow; IPI, International Prognostic Index; FLIPI, follicular lymphoma IPI; WBC, white blood cell count; ALC, absolute lymphocyte count; Hb, hemoglobin; LDH, lactate dehydrogenase; $\beta 2 \mathrm{M}, \beta 2$-microglobulin. 

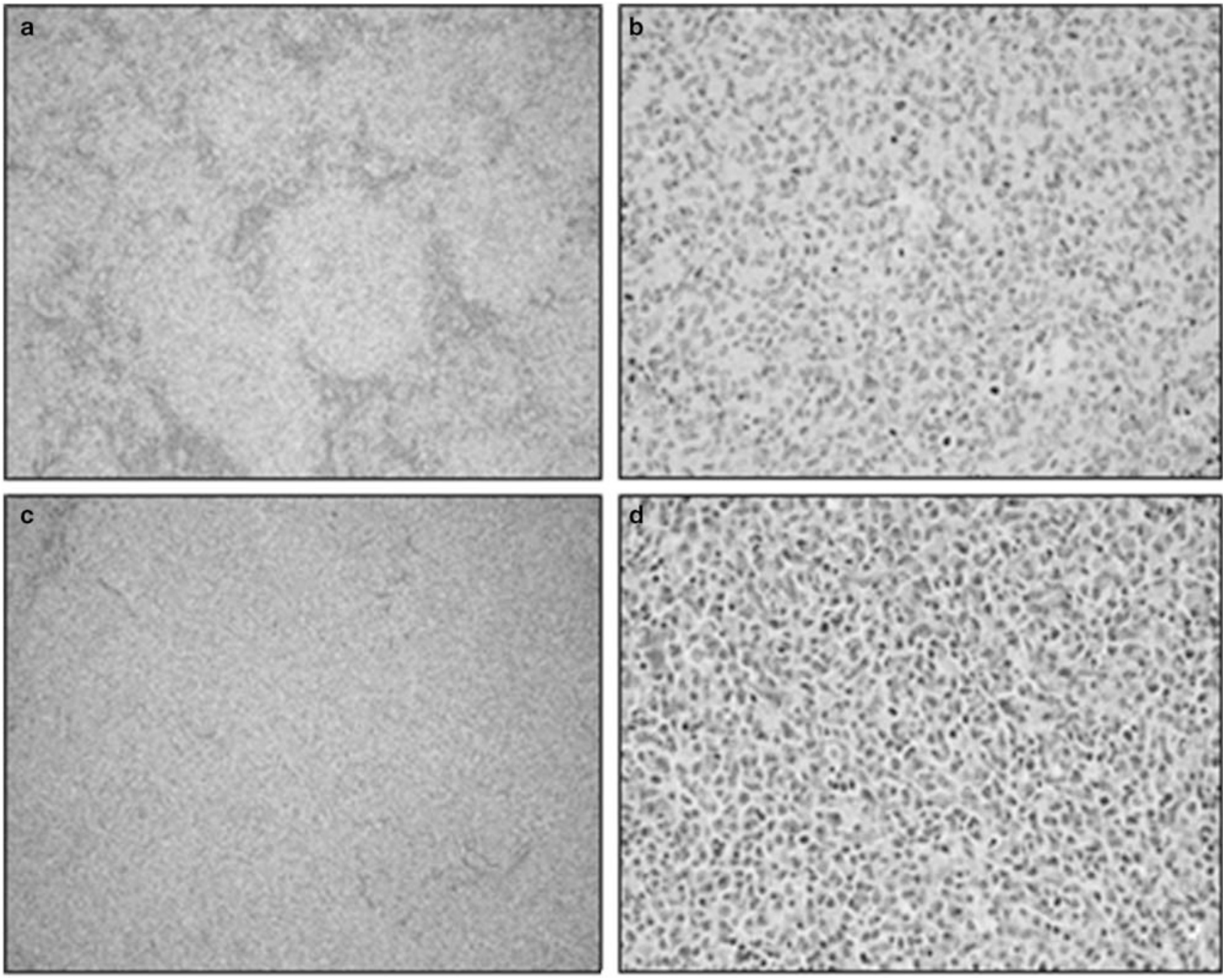

Figure 1 Morphologic features of a case of CD5+ follicular lymphoma involving a cervical lymph node. The lymphoma consists of grade 3B (80-90\%, a,b) and diffuse large B-cell lymphoma (10-20\%, c,d). Immunostains show that the lymphoma cells are positive for CD20 (e), weakly positive for CD5 (f), and are negative for CD3 (g). An antibody for Ki-67 (MIB-1) shows that $\sim 10-60 \%$ of the cells are positive (h). ((a-d) hematoxylin-eosin staining; (a,c) 100X; (b,d) 400X; (e-h) immunohistochemistry with hematoxylin counterstain; e,h 200X; f,g 400X).

population of small-to-medium sized cells with cleaved nuclear contours and scant cytoplasm (centrocytes) and large cells with vesicular chromatin, one to multiple peripheral nucleoli and a narrow rim of cytoplasm (centroblasts) were present. The cellular composition in the areas with a diffuse pattern was similar to that in the follicular areas, except that large cells were relatively less numerous in diffuse areas. Interfollicular monocytoid cells were noted in 3 cases. Focal extension into adjacent adipose tissue was present in some cases. There was no significant difference in the distribution of grades between CD5+ vs CD5- cases. In the CD5+ group, 61 cases were grade 1 or 2 and 27 cases were grade 3 , including 15 grade $3 \mathrm{~A}$ and 12 grade 3B. In the CD5group, 75 cases were grade 1 or 2 and 24 cases were grade 3 , including 14 grade $3 \mathrm{~A}$ and 10 grade $3 \mathrm{~B}$ $(P=0.330)$. One case of CD5+ follicular lymphoma, grade $3 \mathrm{~B}$ associated with diffuse large $\mathrm{B}$-cell lymphoma is shown in Figure 1. A case of CD5+ follicular lymphoma involving bone marrow is shown in Figure 2.

Twenty patients had both CD5+ follicular lymphoma and diffuse large B-cell lymphoma detected concurrently, either in the same $(n=14)$ or different $(n=6)$ biopsy specimens, and 10 patients had grade 3 follicular lymphoma ( 7 cases of grade $3 \mathrm{~A}$ and 3 cases of grade $3 \mathrm{~B}$ ). In another 13 patients, diffuse large B-cell lymphoma developed after the diagnosis of CD5+ follicular lymphoma, with a median interval of 27 months (range, 3-104 months) between the detection of CD5+ follicular lymphoma and the development of diffuse large B-cell lymphoma. Five patients had a diagnosis of diffuse large B-cell lymphoma before the detection of CD5+ follicular lymphoma (median interval, 42 months; range, 29-58 months). In contrast, only 17 of 99 patients 

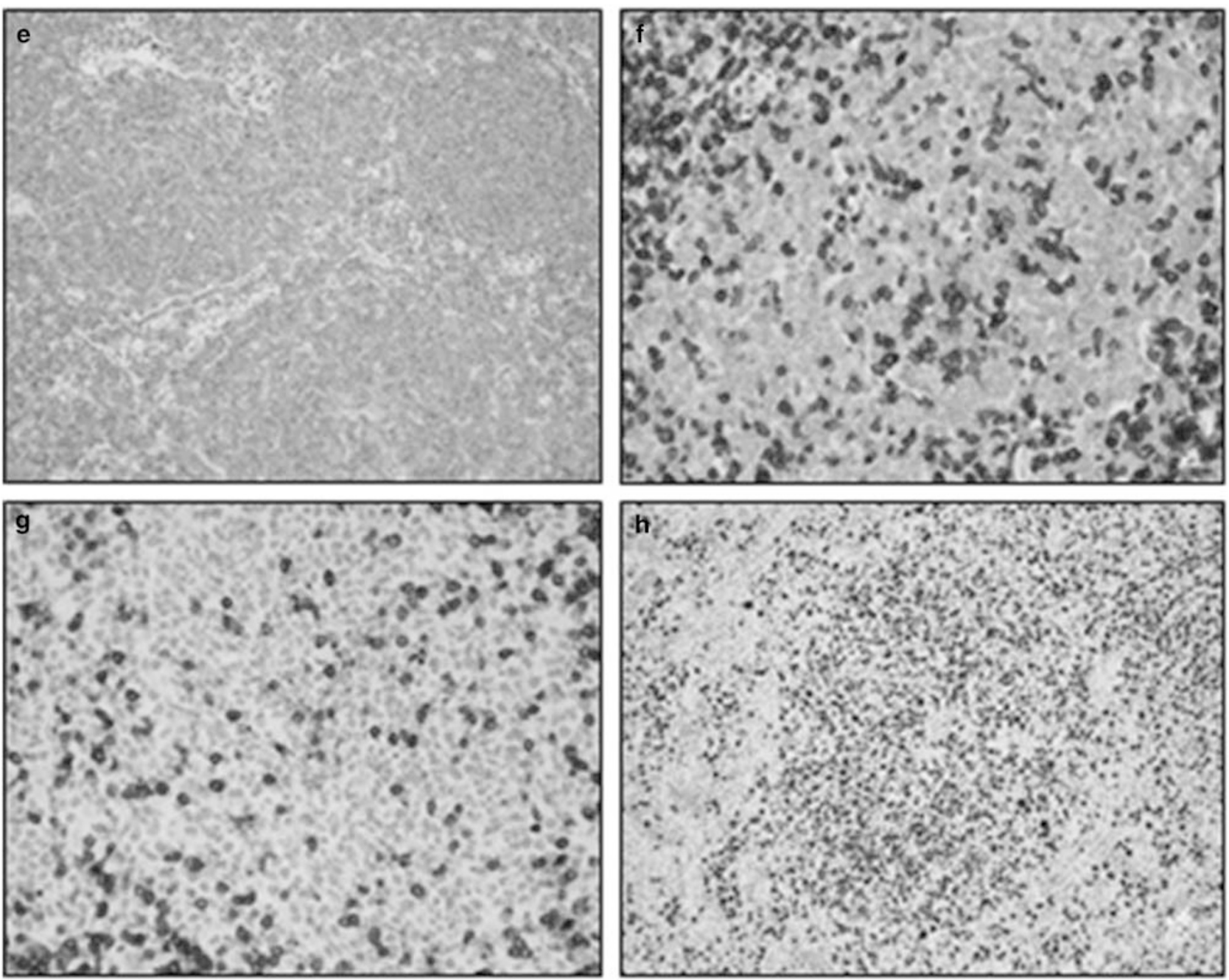

Figure 1 Continued.

in the CD5- follicular lymphoma comparison group developed diffuse large B-cell lymphoma $(P<0.001)$.

\section{Immunophenotypic Findings}

Flow cytometry immunophenotypic analysis was performed on 84 cases. In all cases assessed, the lymphoma cells were positive for CD19 $(n=83)$, CD20 $(n=82)$, CD22 $(n=42)$, CD38 $(n=47)$, and CD79b $(n=16)$, Eighty of $81(99 \%)$ cases assessed expressed CD5, with varying intensity ranging from dim partial $(n=53)$ to moderate $(n=26)$ to bright $(n=1$; Figure 3). CD10 was positive in 57 of $84(68 \%)$ cases, ranging from dim positive $(n=16)$ to moderately-brightly positive $(n=41)$. Other markers expressed in subsets of patients included FMC-7 (47/64, 73\%), CD23 (44/72, 61\%), CD200 (8/20, $40 \%)$, CD43 (10/27, 37\%), CD11c $(6 / 26,23 \%)$, and CD30 $(2 / 11,18 \%)$. Eighty of $83(96 \%)$ cases expressed monotypic surface immunoglobulin light chain (50 kappa, 30 lambda); 3 cases showed aberrant loss of light chain expression. All cases were negative for surface CD3.

Immunohistochemical analysis was performed on 83 cases. All cases assessed were positive for CD20 $(n=68)$, BCL-6 $(n=59)$, and PAX-5 $(n=43)$, and were negative for CD3 $(n=59)$ and cyclin D1 $(n=39)$. CD5 was positive in 19/52 (37\%) cases (including the one case negative by flow cytometry). BCL2 (60/61, 98\%), CD10 (39/54, 72\%), CD30 (3/5, 60\%), MUM1 $(3 / 5,60 \%)$, CD43 (4/12, 33\%), and CD23 (3/11, 27\%) were positive in subsets of cases. Additional immunostains for CD21 $(n=30)$ and CD23 $(n=10)$ revealed follicular dendritic meshworks within follicular areas. Interestingly, in 4 cases CD5 was expressed by centroblasts but not by the centrocytes. The proliferation index, as demonstrated by Ki-67 expression, was assessed on 60 cases. The median Ki-67 index was 30\% (range, 5-75\%). Ki-67 data, sub-divided into $10 \%$ increments, were as follows: $1-10 \% \quad(n=9), 11-20 \% \quad(n=13), 21-30 \% \quad(n=9)$, $31-40 \% \quad(n=7), 41-50 \% \quad(n=11), 51-60 \% \quad(n=6)$, $61-70 \%(n=4)$, and $71-80 \%(n=1)$. 

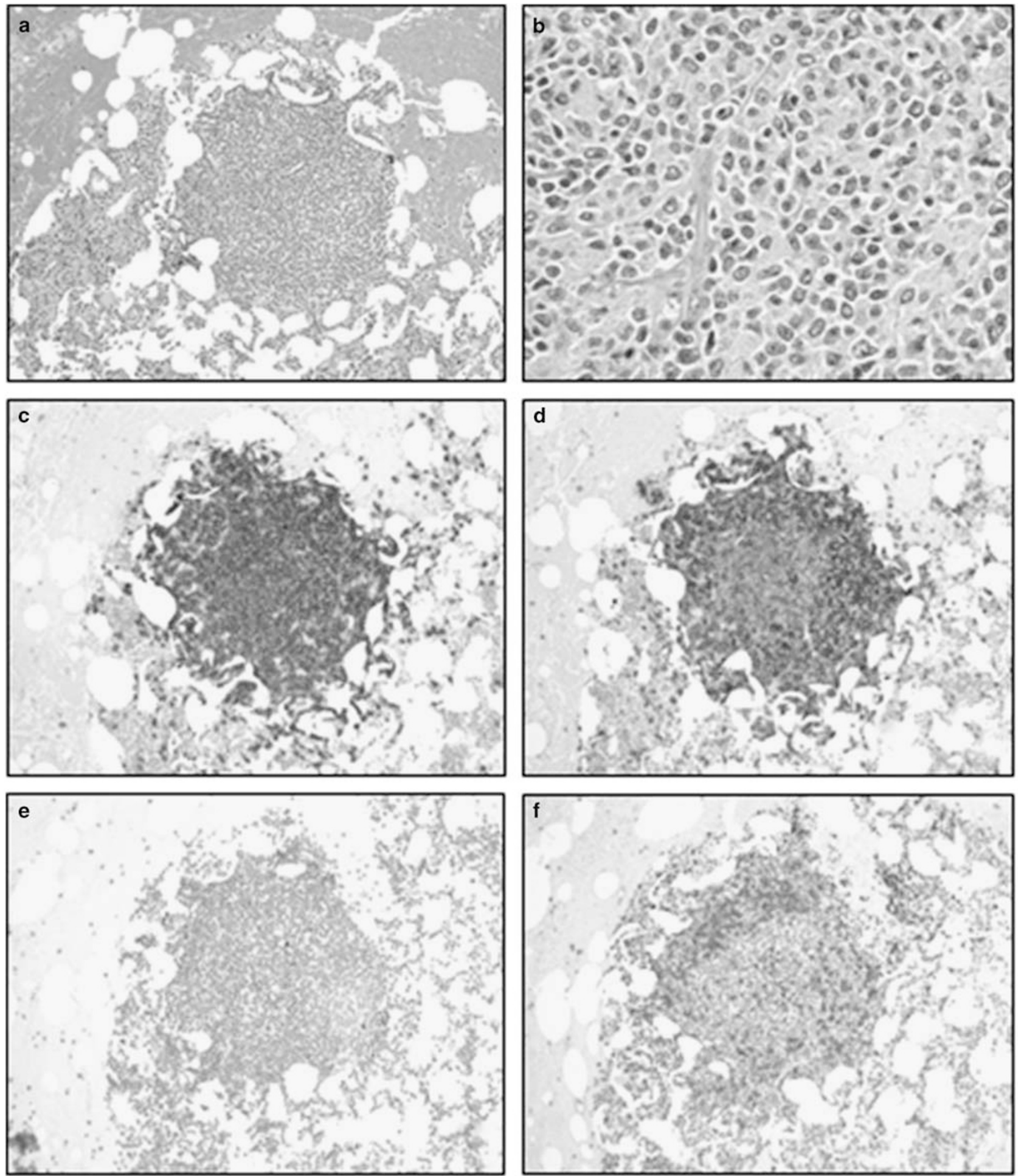

Figure 2 A case of CD5+ follicular lymphoma involving the bone marrow. The lymphoma cells form atypical lymphoid aggregate (a) and show irregular nuclear contours and scant cytoplasm (b). Immunostains show that the lymphoma cells are positive for CD20 (c), CD5 (d) and BCL-6 (e), and are negative for CD3 (f). ((a,b) hematoxylin-eosin; (a) 100X; (b) 400x; (c-f) immunohistochemistry with hematoxylin counterstain, 100X).

Overall, CD5 expression was detected by flow cytometry alone in 69 cases, by immunohistochemistry alone in 8 cases, and by both methods in 11 cases. CD5 was detected at time of initial diagnosis of follicular lymphoma in 60/65 (92\%) patients. In 6 patients, the initial follicular lymphoma was CD5-, but recurrent samples showed CD5 expression. In 22 cases of CD5+ follicular lymphoma, CD5 status in the initial biopsy specimen is unknown. 

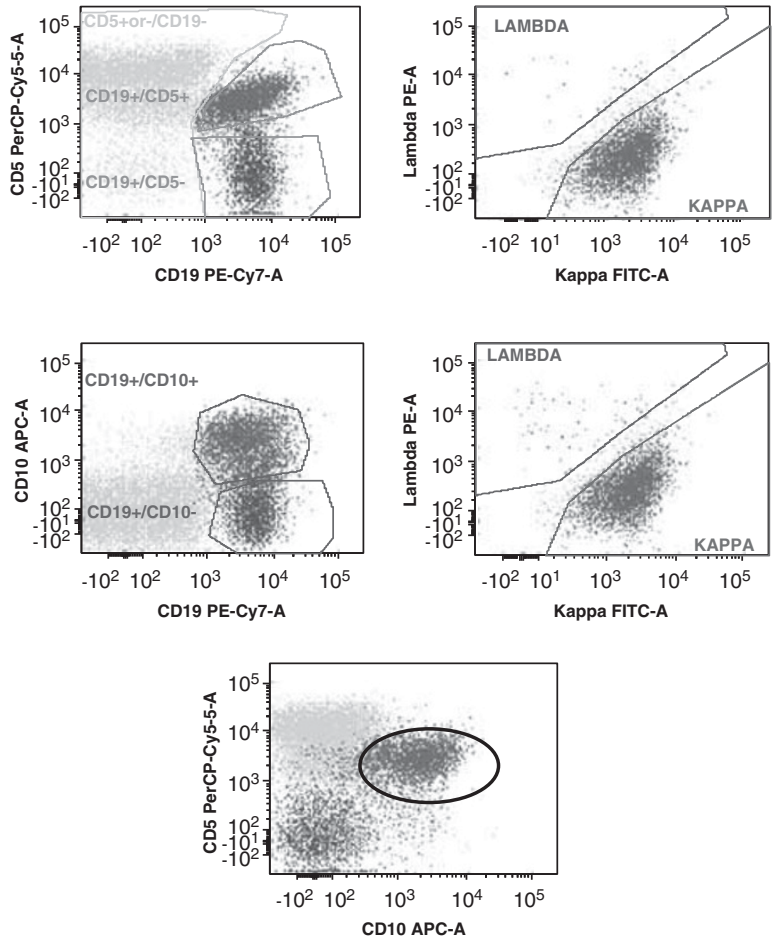

Figure 3 Flow cytometry immunphenotyping of a case of CD5+ follicular lymphoma. Both the CD5+CD19+, and CD10(dim+)CD19 + lymphoma cells show monotypic immunoglobulin kappa light chain restriction.

\section{CD5 Expression in Sequential Biopsy Specimens}

In 31 patients CD5 expression was assessed in more than one biopsy specimen over time. In $21(68 \%)$ patients CD5 expression did not change. In 6 (19\%) patients CD5 expression was negative in the initial biopsy specimen and was acquired subsequently. In 2 of these patients acquisition of CD5 was accompanied by a change in histology from low-grade to grade 3 and in the other 4 cases there was no change in the grade. In the remaining $4(13 \%)$ patients, CD5 was expressed in the first biopsy specimen but was not expressed in the subsequent biopsy specimens. In 2 of these cases, loss of CD5 was accompanied by a change from grade 3 to low-grade and in the other 2 cases there was no change in grade.

\section{Cytogenetic and Molecular Findings}

Conventional cytogenetic analysis was performed on 28 patients. Six cases showed the presence of $\mathrm{t}(14 ; 18)(\mathrm{q} 32 ; \mathrm{q} 21)$ in addition to other abnormalities, 1 case showed $\mathrm{t}(18 ; 22)(\mathrm{q} 21 ; \mathrm{q} 11.2)$ in addition to other abnormalities, 3 cases showed complex karyotypes but without $t(14 ; 18)(q 32 ; q 21)$ or other $18 q 21$ abnormalities, 2 cases showed $-\mathrm{Y}$ as the sole abnormality, and 16 cases had a diploid karyotype. Among the 12 cases with an abnormal karyotype, common cytogenetic aberrations included gains (or partial gains) of chromosomes $\mathrm{X}(n=3), 1 \quad(n=4), 3$ $(n=3), 5(n=4), 7(n=3), 10(n=3)$, and 18q $(n=4)$, and losses (or partial losses) of chromosomes Y $(n=4), 6 q(n=3)$, and $13(n=4)$. Rearrangements of 3q27 occurred in 2 cases (Supplementary Table 1).

Fluorescence in situ hybridization analysis using an $I G H / B C L 2$ translocation probe was performed on 27 cases and showed the presence of the IGH-BCL2 gene rearrangement in 12 cases; 1 case had IGLBCL2. In 10 cases fluorescence in situ hybridization analysis for IGH/CCND1 was negative. Qualitative real-time PCR was performed on 25 cases; 14 cases showed IGH-BCL2 fusion, all involving the major breakpoint region. Overall, the presence of $t(14 ; 18)$ (q32;q21)/IGH-BCL2 or t(18;22)(q21;q11.2)/IGL-BCL2 was detected in 28 of $44(64 \%)$ cases assessed.

\section{Clinical Outcome}

Treatment information was available for 81 patients. Forty-five patients received rituximab-cyclophosphamide, doxorubicin, vincristine, and prednisone (R-CHOP), 26 had other rituximab-based chemotherapy regimens, and 6 received rituximab as a single agent. One of each of the remaining 3 patients received pentostatin, cyclophosphamide, and ordoxorubicin and ifosfamide. One patient received no chemotherapy and was observed. Twelve patients also underwent stem-cell transplantation. With a median follow-up of 55 months (range, 0.5-207 months), 15 patients died; most died of the disease $(n=12)$ whereas others died of therapy-related acute myeloid leukemia $(n=1)$, viral encephalitis $(n=1)$, or unknown cause $(n=1)$. Forty-six patients were alive with disease and 20 patients were in clinical remission at last follow-up. Clinical outcome was not available for 6 patients.

Compared with a group of 99 patients with CD5follicular lymphoma, patients with CD5+ follicular lymphoma had significantly shorter median progression-free survival (44 months vs 89 months, $P=0.0042)$ and 5-year progression-free survival (38\% vs $63.2 \%, P=0.0042$ ). The median overall survival and 5-year overall survival were also shorter in patients with CD5+ follicular lymphoma, but without statistical significance (154 months vs 222 months, $P=0.0793 ; 78.4 \%$ vs $89.8 \%$, $P=0.0793$; Figure 4a). We further sub-divided patients with CD5+ follicular lymphoma into two groups: CD5+ follicular lymphoma with or without diffuse large B-cell lymphoma. There was no statistically significant difference in median overall survival or median progression-free survival between patients who developed diffuse large B-cell lymphoma vs patients who did not develop diffuse large B-cell lymphoma (median overall survival, 156 months in patients with diffuse large B-cell lymphoma and 154 months in patients without diffuse large B-cell lymphoma, $P=0.4761$; median progression-free survival, 31 months in patients with diffuse large B-cell lymphoma and 45 months in 

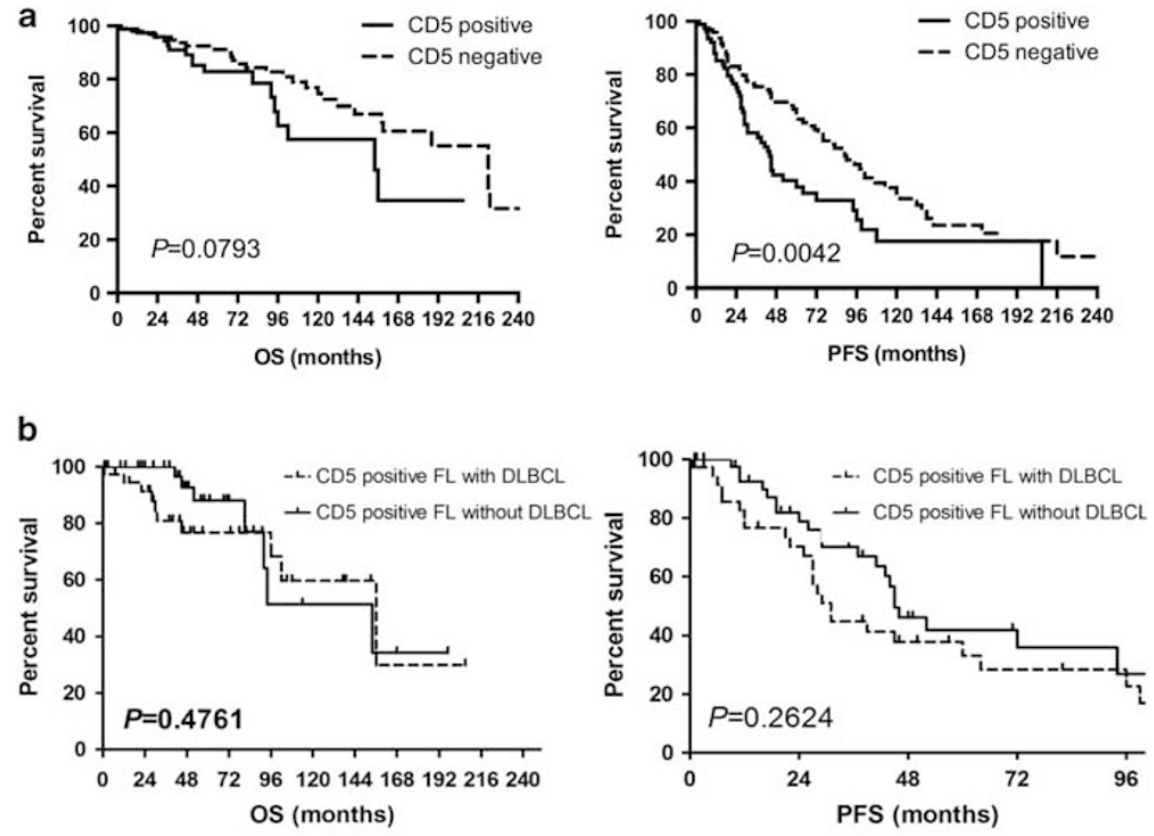

Figure 4 Overall survival and progression-free survival of patients with follicular lymphoma (a) Comparison between patients with CD5+ follicular lymphoma and CD5 - counterpart. (b) Comparison between patients with CD5+ follicular lymphoma with and without diffuse large B-cell lymphoma.

patients without diffuse large B-cell lymphoma, $P=0.2624$; Figure 4b).

Patients with CD5+ follicular lymphoma without diffuse large B-cell lymphoma had shorter progression-free survival $(P=0.0113)$ but not overall survival $(P=0.2396)$ than patients with CD5- follicular lymphoma without diffuse large B-cell lymphoma. There was no significant difference in progression-free survival $(P=0.2802)$ or overall survival $(P=0.7641)$ between patients who had CD5+ follicular lymphoma with diffuse large B-cell lymphoma vs patients who had CD5- follicular lymphoma with diffuse large B-cell lymphoma. In contrast, among patients with CD5- follicular lymphoma, those who had developed diffuse large B-cell lymphoma had a shorter progression-free survival ( $P=0.0002)$, but not overall survival $(P=2561)$, than patients without diffuse large B-cell lymphoma. Moreover, when comparing patients with CD5+ follicular lymphoma without diffuse large B-cell lymphoma vs patients with CD5- follicular lymphoma with diffuse large B-cell lymphoma, we did not observe any significant differences in progression-free survival $(P=0.7778)$ or overall survival $(P=0.4653)$.

\section{Prognostic Factors}

We performed univariate analysis to evaluate the association between 19 clinicopathologic features and prognosis (Table 2). The results showed that a Ki-67 proliferation index of $\geq 60 \%$ and IPI $>2$ were associated with poorer overall survival in both CD5+ and CD5- patients $(P<0.05)$. In the CD5+ group, significantly more patients presented with an IPI $>2$ (35/80) compared with patients in the CD5- group $(10 / 99, P<0.001)$. No significant difference in the number of tumors with Ki-67 $\geq 60 \%$ was observed in the two groups (5/60 in CD5+ group vs 5/83 in CD5group, $P=0.752$ ).

\section{Discussion}

The CD5 antigen is a $67-\mathrm{kDa}$ membrane glycoprotein that is normally found on most mature T-cells and a subset of B-cells in cord blood, adult peripheral blood, and lymph nodes. ${ }^{14}$ In mice, CD5 functions as a negative regulator of cell signaling. ${ }^{15,16}$ In humans, CD5 induces an anergic state in lymphocytes and contributes to B-cell survival and interleukin-10 production, and CD5 expression on B-cells may serve as a marker of antigen exposure. ${ }^{15,16}$

Assessment of CD5 expression by flow cytometry or immunohistochemistry has been widely used in the clinical work-up of B-cell lymphomas. CD5 expression is usually seen in chronic lymphocytic leukemia/small lymphocytic lymphoma or mantle cell lymphoma. ${ }^{17,18}$ However, expression of CD5 also has been detected occasionally in other B-cell lymphomas including extranodal marginal zone lymphoma of mucosa-associated lymphoid tissue, nodal marginal zone lymphoma, splenic marginal zone lymphoma, lymphoplasmacytic lymphoma, follicular lymphoma, and diffuse large B-cell 
Table 2 Univariate analysis of clinical and pathologic features to predict overall and progression-free survival

\begin{tabular}{|c|c|c|c|c|c|c|}
\hline & \multicolumn{3}{|c|}{ Overall survival } & \multicolumn{3}{|c|}{ Progression-free survival } \\
\hline & $\mathrm{P}$ & $H R$ & $95 \%$ CI of $H R$ & $\mathrm{P}$ & $H R$ & $95 \%$ CI of $H R$ \\
\hline Age $(>60$ vs $\leq 60)$ & 0.076 & 2.474 & $0.889-6.889$ & 0.194 & 1.903 & $0.721-5.025$ \\
\hline Gender & 0.398 & 0.646 & $0.235-1.779$ & 0.337 & 0.612 & $0.225-1.668$ \\
\hline B-symptoms & 0.811 & 1.67 & $0.328-4.160$ & 0.65 & 1.335 & $0.383-4.657$ \\
\hline Lymphadenopathy & 0.634 & 0.696 & $0.156-3.101$ & 0.905 & 1.094 & $0.249-4.805$ \\
\hline Splenomegaly & 0.45 & 1.645 & $0.452-5.987$ & 0.075 & 3.175 & $0.888-11.345$ \\
\hline Nodal vs extranodal & 0.886 & 0.926 & $0.325-2.640$ & 0.974 & 0.982 & $0.343-2.816$ \\
\hline BM involvement & 0.254 & 1.938 & $0.622-6.039$ & 0.159 & 2.268 & $0.726-7.091$ \\
\hline Stage III/IV vs stage I/II & 0.472 & 1.723 & $0.390-7.606$ & 0.525 & 1.617 & $0.367-7.130$ \\
\hline IPI $(>2 v s \leq 2)$ & 0.034 & 3.175 & $1.089-9.252$ & 0.062 & 2.588 & $0.954-7.022$ \\
\hline FLIPI $(>2 v s \leq 2)$ & 0.304 & 1.368 & $0.753-2.487$ & 0.382 & 1.291 & $0.728-2.290$ \\
\hline WBC $(<4 \mathrm{k} / \mu \mathrm{l}$ vs $4-11$ vs $>11 \mathrm{k} / \mu \mathrm{l})$ & 0.056 & 3.154 & $0.957-10.400$ & 0.241 & 1.944 & $0.640-5.908$ \\
\hline $\operatorname{ALC}(<1000$ vs $\geq 1000 / \mu \mathrm{l})$ & 0.615 & 0.77 & $0.278-2.131$ & 0.938 & 0.961 & $0.355-2.606$ \\
\hline $\mathrm{Hb}(<12$ vs $\geq 12 \mathrm{~g} / \mathrm{dl})$ & 0.261 & 2.066 & $0.583-7.328$ & 0.028 & 4.347 & $1.176-16.069$ \\
\hline Platelet $(<140 v s \geq 140 \mathrm{k} / \mu \mathrm{l})$ & 0.145 & 2.131 & $0.770-5.901$ & 0.125 & 2.195 & $0.803-5.996$ \\
\hline LDH (high vs normal) & 0.177 & 2.013 & $0.729-5.561$ & 0.222 & 1.869 & $0.684-5.106$ \\
\hline$\beta 2 \mathrm{M}$ (high vs normal) & 0.676 & 0.784 & $0.249-2.464$ & 0.917 & 0.941 & $0.299-2.960$ \\
\hline Grade (III vs I/II) & 0.42 & 1.505 & $0.557-4.065$ & 0.479 & 1.435 & $0.529-3.893$ \\
\hline Ki-67 ( $\geq 60 \%$ vs $<60 \%)$ & 0.027 & 3.719 & $1.158-11.944$ & 0.061 & 2.995 & $0.951-9.428$ \\
\hline Large B-cell lymphoma & 0.234 & 1.836 & $0.675-4.992$ & 0.202 & 1.917 & $0.705-5.212$ \\
\hline
\end{tabular}

Abbreviations: HR, hazard ratio; CI, confidence interval; BM, bone marrow; IPI, international prognostic index; FLIPI, follicular lymphoma IPI; WBC, white blood cell count; ALC, absolute lymphocyte count; Hb, hemoglobin; LDH, lactate dehydrogenase; $\beta 2 \mathrm{M}, \beta 2$-microglobulin.

Table 3 Summary of previously reported cases of CD5+ follicular lymphoma

\begin{tabular}{|c|c|c|c|c|c|c|c|c|c|c|}
\hline Author/year & $\begin{array}{l}\text { No. of } \\
\text { cases }\end{array}$ & Gender & Site & Grade & CD10- & CD43+ & $B M$ & $t(14 ; 18)$ & Treatment & Outcome (follow up) \\
\hline Tiesigna/2000 & 4 & $3 \mathrm{M} / 1 \mathrm{~F}$ & Nodal & $\begin{array}{c}1-2 \\
\text { Floral }\end{array}$ & $1 / 4$ & NA & $3 / 4$ & 4 & Chemo & $\begin{array}{l}3 \text { alive }(15-42 \mathrm{~m}) \\
1 \text { died }(50 \mathrm{~m})\end{array}$ \\
\hline Barekman/2001 & 1 & $\mathrm{~F}$ & Scalp & 1 & $0 / 1$ & NA & NA & NA & $\mathrm{rxt}$ & Alive $(5 \mathrm{~m})$ \\
\hline Barry/2002 & 3 & $2 \mathrm{M} / 1 \mathrm{~F}$ & Nodal & $1,2,3 \mathrm{~A}$ & $0 / 3$ & $0 / 3$ & $2 / 3$ & $2 / 3$ & None & NA \\
\hline Dong/2003 & 10 & NA & Nodal $^{\mathrm{a}}$ & $\begin{array}{c}1-2(9) \\
3+\text { LBCL (1) }\end{array}$ & $0 / 10$ & $0 / 10$ & NA & NA & Chemo (1) & $\begin{array}{l}\text { Persistent } \\
\text { disease (2y) }\end{array}$ \\
\hline Sekiguchi/2011 & 1 & $\mathrm{M}$ & Nodal & $3 \mathrm{~A}$ & $0 / 1$ & NA & NA & $0 / 1$ & Chemo & CR $(4 \mathrm{~m})$ \\
\hline Mayson/2014 & 2 & $2 \mathrm{~F}$ & Nodal & 1 & $0 / 2$ & NA & $2 / 2$ & $1 / 1$ & Chemo & $\begin{array}{l}1 \text { CR (NA) } \\
1 \text { persistent } \\
\text { disease (NA) }\end{array}$ \\
\hline Miyoshi/2014 & 22 & $12 \mathrm{M} / 10 \mathrm{~F}$ & $\begin{array}{l}\text { Nodal (12) } \\
\text { Extranodal (4) } \\
\text { Unknown (6) }\end{array}$ & $\begin{array}{c}1-2(11) \\
3(11)\end{array}$ & $4 / 22$ & NA & $4 / 16$ & $9 / 20$ & $\begin{array}{l}\text { Chemo+/ - rxt (16) } \\
\text { None (1) }\end{array}$ & $\begin{array}{l}\text { CR }(13 / 16) \\
32 \mathrm{~m}(1.5-114 \mathrm{~m})\end{array}$ \\
\hline Current & 88 & $53 \mathrm{M} / 35 \mathrm{~F}$ & $\begin{array}{l}\text { Nodal (66) } \\
\text { Extranodal (22) }\end{array}$ & $\begin{array}{c}1-2(61) \\
3(27) \\
\text { LBCL }(38)^{b}\end{array}$ & $27 / 84$ & $10 / 27$ & $46 / 78$ & $28 / 44$ & $\begin{array}{l}\text { Chemo (81/81) } \\
12 \text { (add sct) }\end{array}$ & $\begin{array}{l}20 \mathrm{CR}, 46 \text { persistent } \\
\text { disease; } 15 \text { died } \\
55 \mathrm{~m}(0.5-207 \mathrm{~m})\end{array}$ \\
\hline
\end{tabular}

Abbreviations: M, male; F, female; NA, not available; LBCL, large B-cell lymphoma; BM, bone marrow; chemo, chemotherapy; rxt, radiation therapy; add sct, additional stem-cell transplant; CR, clinical remission.

${ }^{a}$ All 10 cases involved lymph nodes, 2 cases also involved skin.

barge B-cell lymphoma developed prior to, at the same time, or after follicular lymphoma.

lymphoma. ${ }^{9,19-23}$ In follicular lymphoma, CD5 expression has been associated with younger age, male predominance and grade 3 morphology. $4,6,8$ More recently, Miyoshi et al ${ }^{10}$ in Japan reported a series of 22 cases of CD5+ follicular lymphoma and found that patients with CD5+ follicular lymphoma showed a higher frequency of peripheral blood involvement, CD25 and MUM1 expression, and a lower frequency of $t(14 ; 18)(\mathrm{q} 32 ; \mathrm{q} 21$; Table 3$)$. In this study, we describe the clinicopathologic, immunophenotypic, and molecular genetic features of 88 cases of CD5+ follicular lymphoma from the United
States, the largest series to date. As these cases represent $2.7 \%$ of all follicular lymphomas seen in our institution within the study period, we confirm that CD5 expression in follicular lymphoma is rare, but likely is a marker of a distinctive entity that needs to be considered in the differential diagnoses of CD5+ B-cell lymphomas.

Unlike CD5- follicular lymphoma, patients with CD5+ follicular lymphoma have several distinct clinicopathologic features. Men are affected more often than women with a male-to-female ratio of 1.5 to 1 (this ratio is closer to $1: 1$ in CD5- follicular 
lymphoma). CD5+ follicular lymphoma tends to occur more frequently at extranodal sites (25\%), with a predilection for the head and neck region and the gastrointestinal tract. In this study group, over $90 \%$ of patients had more than one involved site and $84 \%$ of patients presented with stage III or IV disease, higher than the reported frequency of $67 \%$ for patients with CD5- follicular lymphoma. ${ }^{24}$ Others have reported that $80-90 \%$ of follicular lymphomas are grade 1 or 2 lesions. ${ }^{1}$ However, $31 \%$ of cases in our series were grade 3 . Lastly, it has been reported that $25-35 \%$ of patients with follicular lymphoma may undergo histologic transformation to diffuse large B-cell lymphoma and transformation is usually associated with a rapidly progressive clinical course, refractoriness to therapy and death. ${ }^{1,25}$ In our series, a much higher percentage of patients $(43 \%)$ developed diffuse large B-cell lymphoma, whereas only $17 \%$ patients from the CD5- follicular lymphoma group developed diffuse large B-cell lymphoma $(P<0.001)$. Finally, when compared with a group of patients with CD5- follicular lymphoma matched for age, gender, and stage, we found that patients with CD5+ follicular lymphoma had a significantly shorter median progression-free survival.

The clinical course of follicular lymphoma is highly variable. Important adverse prognostic indicators include older age at diagnosis, advanced stage, and high proliferation rate (as demonstrated by assessing Ki-67), histologic transformation, high serum levels of LDH and $\beta 2$-microglobulin, complex molecular genetic aberrations, and high IPI. ${ }^{26}$ Some reports have suggested that CD5+ follicular lymphoma may be prone to undergo transformation and thus associated with a poorer prognosis. ${ }^{27}$ Miyoshi et $a l^{10}$ recently also reported that CD5 expression was an independent poor prognosis factor in patients with follicular lymphoma. The results we report show that patients with CD5+ follicular lymphoma have significant shorter median progression-free survival and 5-year progression-free survival than patients with CD5- follicular lymphoma. The median overall survival and 5-year overall survival were also shorter for patients with CD5+ follicular lymphoma, although not statistically significant, possibly related to the sample size of this study. Surprisingly, among patients with CD5+ follicular lymphoma, we did not observe any statistical significance in overall survival or progression-free survival. We do not have an explanation for this finding; however, one possibility may be that the poor prognostic effect of large cell transformation on survival is mitigated by the adverse prognostic impact of CD5 expression. The observation that no significant difference exists in progression-free survival and overall survival between patients with CD5+ follicular lymphoma without diffuse large B-cell lymphoma vs CD5follicular lymphoma with diffuse large B-cell lymphoma further supports this possibility.
The data presented here suggest that CD5+ follicular lymphoma has several different immunophenotypic features compared with its CD5- counterpart. Flow cytometry immunophenotyping showed that about one third of CD5+ follicular lymphoma cases assessed were positive for CD43 or CD200 $(\sim 40 \%)$ or were negative for CD10. CD43 expression is unusual in follicular lymphoma, but has not been previously associated with CD5 expression. No data has been reported regarding CD200 expression in CD5+ follicular lymphoma. Others have reported that follicular lymphoma can be CD10-negative, more common in grade $3 \mathrm{~B}$ cases. ${ }^{28,29}$ Our results differ somewhat in that 11 of $27(41 \%)$ cases that lacked CD10 were low grade. Lack of CD10 expression in follicular lymphoma also has been associated with absence of IGH/BCL2 translocation. ${ }^{29}$ Among 19 cases that lacked CD10 expression in this study, we detected IGH/BCL2 gene rearrangement in 8 $(42 \%)$ cases, a rate that was higher than the $5 \%$ reported by Karube et al, ${ }^{29}$ but was lower than the $85-90 \%$ reported in the CD5- follicular lymphoma.

The genetic hallmark of follicular lymphoma, $\mathrm{t}(14 ; 18)(\mathrm{q} 32 ; \mathrm{q} 21) / I G H-B C L 2$, is present in $80-90 \%$ of follicular lymphoma, depending on the method used to detect this abnormality, with fluorescence in situ hybridization being the most sensitive method. ${ }^{2}$ Geographic variation between Western and Asian populations also has been reported. ${ }^{30}$ Moreover, $B C L 2$ rearrangements are much less frequent in grade 3B follicular lymphoma. ${ }^{31}$ Using conventional cytogenetic analysis, fluorescence in situ hybridization and polymerase chain reaction-based assays, we detected $\mathrm{t}(14 ; 18)(\mathrm{q} 32 ; \mathrm{q} 21) / I G H-B C L 2$ in $28 / 44(64 \%)$ of CD5+ follicular lymphoma, which may reflect the presence of a higher frequency of grade 3 lesions and diffuse large B-cell lymphoma in our series.

In addition to the $t(14 ; 18)(\mathrm{q} 32 ; \mathrm{q} 21) / I G H-B C L 2$, other genetic aberrations have been reported in $\sim 90 \%$ of follicular lymphomas and most commonly include losses of chromosomes 1p, 6q, 10q, and 17p, and gains of chromosomes 1, 6p, 7, 8, 12q, X, and $18 \mathrm{q}{ }^{32-34}$ Abnormalities of $3 \mathrm{q} 27$ and/or BCL-6 rearrangement are found in $5-15 \%$ of follicular lymphoma. ${ }^{35}$ The number of additional cytogenetic abnormalities increases with increasing histologic grade or histologic transformation to diffuse large B-cell lymphoma. ${ }^{36}$ Bouska et $a l^{37}$ recently compared 198 cases of follicular lymphoma and 79 cases of transformed follicular lymphoma using a singlenucleotide polymorphism array to identify secondary chromosomal abnormalities that drive the development of follicular lymphoma and its transformation to diffuse large B-cell lymphoma. The authors found that common recurrent chromosomal abnormalities in follicular lymphoma included gains of $2 p, 5,6 p, 7$, $8,12 q, 17 q, 18,21$, and $X$, and losses of $1 p, 6 q, 10 q$, and $17 \mathrm{p} .{ }^{37}$ In addition, Bouska et $a l^{37}$ found that gains of 3q27.3-q28 and chromosome 11 and losses of 9p21.3 and 15q were more frequent in transformed 
follicular lymphoma. In keeping with these findings, among 12 cases of CD5+ follicular lymphoma with an abnormal karyotype, we found common cytogenetic aberrations including gains of chromosomes X, $1,3,5,7,10$, and 18q, and losses of chromosomes $Y$, $6 q$, and 13. Rearrangements of 3q27 were observed in 2 cases. Furthermore, the presence of more than 6 chromosomal aberrations and a complex karyotype has been correlated with a poor clinical outcome, and $1 p-$, del6q23-26, $+12,17 p-,+18 p$, and $+X p$ have been associated with poor prognosis. ${ }^{33,36,37}$ Most of our CD5+ follicular lymphoma cases had very complex karyotypes, which may account for the higher rate of diffuse large B-cell transformation and poor clinical outcome.

The expression of CD5 in follicular lymphoma raises a differential diagnosis with other CD5+ B-cell lymphomas, in particularly chronic lymphocytic leukemia/small lymphocytic lymphoma, mantle cell lymphoma, and less commonly CD5+ marginal zone lymphoma and CD5+ lymphoplasmacytic lymphoma. Despite some atypical immunophenotypic features, all cases in this series demonstrated classic histologic features of follicular lymphoma, with all but one case showing a follicular (at least partial) pattern and all cases were composed of centrocytes and centroblasts. The case with a diffuse pattern was a small needle biopsy specimen; the lymphoma cells showed a typical follicular lymphoma immunophenotype (CD10 dim positive, CD19+, CD20+, BCL2-, BCL-6+). In all cases, a final diagnosis was rendered based on a combination of histological, immunophenotypic, and cytogenetic findings. None of the cases showed prominent proliferation centers or the characteristic immunophenotype of chronic lymphocytic leukemia/small lymphocytic lymphoma by flow cytometry (ie, dim CD20 and dim immunoglobulin light chain expression) except the case of composite CD5+ follicular lymphoma and chronic lymphocytic leukemia/small lymphocytic lymphoma which had a follicular lymphoma clone and a chronic lymphocytic leukemia/small lymphocytic lymphoma clone. All cases assessed were negative for cyclin D1 and/or IGH/CCND1 fusion, excluding the diagnosis of mantle cell lymphoma. None of these patients assessed had a serum IgM paraprotein characteristic of lymphoplasmacytic lymphoma/ Waldenstrom macroglobulinemia. $\mathrm{t}(14 ; 18)(\mathrm{q} 32 ; \mathrm{q} 21) /$ $I G H-B C L 2$ or other BCL2 translocation was present in 28 cases and over $90 \%$ of cases assessed had other cytogenetic aberrations that are often seen in follicular lymphoma, additional evidence supporting the diagnosis of follicular lymphoma.

In summary, the results of this study show that CD5+ follicular lymphoma is uncommon $(<3 \%)$ and is often associated with involvement by multiple sites, presentation at a more advanced stage, lower frequency of $I G H / B C L 2$ gene rearrangement, higher IPI, a higher rate of transformation to diffuse large B-cell lymphoma, and a shorter progression-free survival.

\section{Disclosure/conflict of interest}

The authors declare no conflict of interest.

\section{References}

1 Harris NL, Nathwani BN, Swerdlow SH et al. Follicular lymphoma In: Swerdlow SH, Campo E, Harris NL, Jaffe ES, Pileri SA, Stein H, Thiele J, Vardiman JW (eds). WHO Classification of Tumours of Haematopoietic and Lymphoid Tissues. IARC: Lyon, 2008;220-226.

2 Horsman DE, Gascoyne RD, Coupland RW et al. Comparison of cytogenetic analysis, southern analysis, and polymerase chain reaction for the detection of $t$ $(14 ; 18)$ in follicular lymphoma. Am J Clin Pathol 1995;103:472-478.

3 Dorfman DM, Shahsafaei A. Usefulness of a new CD5 antibody for the diagnosis of T-cell and B-cell lymphoproliferative disorders in paraffin sections. Mod Pathol 1997;10:859-863.

4 Tiesinga JJ, Wu CD, Inghirami G. CD5+ follicular center lymphoma: immunophenotyping detects a unique subset of "floral" follicular lymphoma. Am J Clin Pathol 2000;114:912-921.

5 Barekman CL, Aguilera NS, Abbondanzo SL. Lowgrade B-cell lymphoma with coexpression of both CD5 and CD10. A report of 3 cases. Arch Pathol Lab Med 2001;125:951-953.

6 Barry TS, Jaffe ES, Kingma DW et al. CD5+ follicular lymphoma: a clinicopathologic study of three cases. Am J Clin Pathol 2002;118:589-598.

7 Dong HY, Gorczyca W, Liu Z et al. B-cell lymphomas with coexpression of CD5 and CD10. Am J Clin Pathol 2003;119:218-230.

8 Sekiguchi Y, Imai H, Wakabayashi $\mathrm{M}$ et al. CD5positive follicular lymphoma: a case report and literature review. Intern Med 2011;50:899-904.

9 Mayson E, Saverimuttu J, Cartwright K. CD5-positive follicular lymphoma: prognostic significance of this aberrant marker? Intern Med J 2014;44:417-422.

10 Miyoshi H, Sato K, Yoshida M et al. CD5-positive follicular lymphoma characterized by CD25, MUM1, low frequency of $t(14 ; 18)$ and poor prognosis. Pathol Int 2014;64:95-103.

11 Yin CC, Lin P, Carney DA et al. Chronic lymphocytic leukemia/small lymphocytic lymphoma associated with IgM paraprotein. Am J Clin Pathol 2005;123: 594-602.

12 Yin CC, Medeiros LJ, Cromwell CC et al. Sequence analysis proves clonal identity in five patients with typical and blastoid mantle cell lymphoma. Mod Pathol 2007;20:1-7.

13 Yin CC, Luthra. R. Molecular detection of t(14;18)(q32; q21) in follicular lymphoma. Methods Mol Biol 2013; 999:203-209.

14 Youinou P, Jamin C, Lydyard PM. CD5 expression in human B-cell populations. Immunol Today 1999;20: 312-316.

15 Gary-Gouy H, Bruhns P, Schmitt C et al. The pseudoimmunoreceptor tyrosin-based activation motif of CD5 mediates its inhibitory action on B-cell receptor signaling. J Biol Chem 2000;275:546-556.

16 Carsetti R, Rosado MM, Wardemann H. Peripheral development of $\mathrm{B}$ cells in mouse and man. Immunol Rev 2004;197:179-191. 
17 Chen CC, Raikow RB, Sonmez-Alpan E et al. Classification of small B-cell lymphoid neoplasms using a paraffin section immunohistochemical panel. Appl Immunohistochem Mol Morphol 2000;8:1-11.

18 Jevermovic D, Dronca RS, Morice WG et al. CD5+ B-cell lymphoproliferative disorders: beyond chronic lymphocytic leukemia and mantle cell lymphoma. Leuk Res 2010;34:1235-1238.

19 Konoplev S, Medeiros LJ, Bueso-Ramos CE et al. Immunophenotypic profile of lymphoplasmacytic lymphoma/Waldenstrom macroglobulinemia. Am J Clin Pathol 2005;124:414-420.

20 Jaso J, Chen L, Li S et al. CD5-positive mucosa associated lymphoid tissue (MALT) lymphoma: a clinicopathologic study of 14 cases. Hum Pathol 2012;43:1436-1443.

21 Jaso JM, Yin CC, Wang SA et al. Clinicopathologic features of CD5-positive nodal marginal zone lymphoma. Am J Clin Pathol 2013;140:693-700.

22 Baseggio L, Traverse-Glehen A, Petinataud F et al. CD5 expression identifies a subset of splenic marginal zone lymphomas with higher lymphocytosis: a clinicopathological, cytogenetic and molecular study of 24 cases. Haematologica 2010;95:604-612.

23 Yamaguchi M, Nakamura N, Suzuki R et al. De novo CD5+ diffuse large B-cell lymphoma: results of a detailed clinicopathological review in 120 patients. Haematologica 2008;93:1195-202.

24 The Non-Hodgkin's Lymphoma Classification Project. A clinical evaluation of the International Lymphoma Study Group classification of non-Hodgkin's lymphoma.. Blood 1997;89:3909-18.

25 Bastion Y, Sebban C, Berger F et al. Incidence, predictive factors, and outcome of lymphoma transformation in follicular lymphoma patients. J Clin Oncol 1997;15:1587-1594.

26 Gallagher CJ, Gregory WM, Jones AE et al. Follicular lymphoma: prognostic factors for response and survival. J Clin Oncol 1986;4:1470-1480.
27 Manazza AD, Bonello L, Pagano $\mathrm{M}$ et al. Follicular origin of a subset of CD5+ diffuse large B-cell lymphomas. Am J Clin Pathol 2005;124:182-190.

28 Marafioti T, Copie-Bergman C, Calaminici M et al. Another look at follicular lymphoma: immunophenotypic and molecular analyses identify distinct follicular lymphoma subgroups. Histopathology 2013;62:860-875.

29 Karube K, Guo Y, Suzumiya J et al. CD10-Mum1+ follicular lymphoma lacks BCL2 gene translocation and shows characteristic biologic and clinical features. Blood 2007;109:306-309.

30 Biagi JJ, Seymour JF. Insights into the molecular pathogenesis of follicular lymphoma arising from analysis of geographic variation. Blood 2002;99:4265-4275.

31 Ott G, Katzenberger T, Lohr A et al. Cytomorphologic, immunohistochemical, and cytogenetic profiles of follicular lymphoma: 2 types of follicular lymphoma grade 3. Blood 2002;99:3806-3912.

32 Offit K, Parsa NZ, Gaidano G et al. 6q deletions define distinct clinic-pathologic subsets of non-Hodgkin's lymphoma. Blood 1993;82:2157-2162.

33 Tilly H, Rossi A, Stamatoullas A et al. Prognostic value of chromosomal abnormalities in follicular lymphoma. Blood 1994;84:1043-1049.

34 Hoglund M, Sehn L, Connors JM et al. Identification of cytogenetic subgroups and karyotypic pathways of clonal evolution in follicular lymphomas. Genes Chromosomes Cancer 2004;39:195-204.

35 Katzenberger $\mathrm{T}$, Ott G, Klein $\mathrm{T}$ et al. Cytogenetic alterations affecting BCL6 are predominantly found in follicular lymphomas grade $3 \mathrm{~B}$ with a diffuse large B-cell component. Am J Pathol 2004;165:481-490.

36 Levine EG, Arthur DC, Frizzera G et al. Cytogenetic abnormalities predict clinical outcome in non-Hodgkin lymphoma. Ann Intern Med 1988;108:14-20.

37 Bouska A, Mckeithan TW, Deffenbacher KE et al. Genome-wide copy-number analyses reveal genomic abnormalities involved in transformation of follicular lymphoma. Blood 2014;123:1681-1690.

Supplementary Information accompanies the paper on Modern Pathology website (http://www.nature.com/ modpathol) 